\title{
Enhancement of Stabilization Control in Remote Robot System with Force Feedback
}

\author{
Pingguo Huang1, Takanori Miyoshi², Yutaka Ishibashi ${ }^{3}$ \\ ${ }^{1}$ Seijoh University, Tokai, Japan \\ ${ }^{2}$ Nagaoka University of Technology, Nagaoka, Japan \\ ${ }^{3}$ Nagoya Institute of Technology, Nagoya, Japan \\ Email: huangpg@seijoh-u.ac.jp, miyoshi@vos.nagaokaut.ac.jp, ishibasi@nitech.ac.jp
}

How to cite this paper: Huang, P.G. Miyoshi, T. and Ishibashi, Y. (2019) Enhancement of Stabilization Control in Remote Robot System with Force Feedback. Int. J. Communications, Network and System Sciences, 12, 99-111.

https://doi.org/10.4236/ijcns.2019.127008

Received: May 27, 2019

Accepted: July 15, 2019

Published: July 18, 2019

Copyright (c) 2019 by author(s) and Scientific Research Publishing Inc. This work is licensed under the Creative Commons Attribution International License (CC BY 4.0).

http://creativecommons.org/licenses/by/4.0/

\begin{abstract}
In this paper, we deal with a remote robot system in which a user can operate an industrial robot with a force sensor at a remote location by using a haptic interface device. We apply a method using the wave filter together with the phase control filter which was previously proposed by the authors to the remote robot system for stabilization control. We also propose a method to enhance the haptic quality. By experiment, we demonstrate the effectiveness of the proposed method. We compare the proposed method with the conventional method quantitatively and clarify which domains the proposed method is applied to more effectively.
\end{abstract}

\section{Keywords}

Remote Robot System, Stabilization Control, Force Feedback, Quality, Experiment

\section{Introduction}

Recently, a number of researchers focus on studies of remote robot systems with force feedback [1] [2] [3]. By using force feedback, since users can touch remote objects and feel the shape, weight, and softness of the objects, the efficiency and accuracy are expected to be largely improved [4]. However, when the haptic information such as position and/or force information is transmitted over a Quality of Sevice (QoS) [5] non-guaranteed network like the Internet, QoS may seriously deteriorate [3] [4]. Furthermore, in the remote robot systems, when the network delay increases, the reaction force becomes larger, and unstability phenomena such as vibrations of the robot and device may occur [6] [7] [8]. To solve the problems, we need to carry out stabilization control and QoS control together [4]. 
There are many studies which focus on remote roboot systems with force feedback [9] [10]. In [9], the authors asssess the Quality of Experience (QoE) [11] about the operability of a haptic interface device for work in which a user operates an industrial robot with a force sensor at a remote location by using the haptic interface device while watching video. They investigate the influence of network delay on the operability and the effect of the adaptive reaction force control [12]. As a result, the control is effective for the remote robot system with force feedback. In [10], Banthia et al. make a prototype telerobotic system for live transmission line maintenance. In the system, a user can operate a haptic interface device at a local terminal to control a remote robot arm. Then, the user tries to do the maintenance by controlling the remote robot arm. They also invetigate the difference in force feedback control (unilateral or bilateral control) by experiment. However, in the papers, they do not carry out stabilization control for the remote robot systems with force feedback. On the otherhand, Miyoshi et al. propose stabilization control which uses the wave filter together with the phase control filter for a haptic telecontrol system in which 1-DoF haptic interface devices are employed in [13] and [14].

In this paper, we mainly focus on the stabilization control. In a preliminaly experiment, we applied out the wave filter together with the phase control filter to the remote robot system with force feedback for stabilization control, and investigated the effect of the stabilization control. In order to clarify the effect of the conventional stabilization control, we pushed a soft object (rubber ball). As a result, we found that the control is effective [7]. However, we found that the control is less effective for hard objects. Therefore, we propose a method in which we incorporate a phase lead compensation filter and a phase lag compensation filter with the conventional control in [7] to achieve stabilization and enhance the haptic quality and investigate the effect of the proposed method and clarify the effective domains by comparing the proposed method with the conventional method. This paper has the following two main contributions: 1) we propose an enhancement method of stabilization control to improve the haptic quality; 2) we also clarify which domains the proposed method and conventional method are applied to more effectively.

The remainder of this paper is organized as follows. Section 2 describes the remote robot system with force feedback. Section 3 introduces the conventional stabilization control and the proposed method. Section 4 explains assessment methods, and assessment results are presented in Section 5. Finally, Section 6 concludes the paper.

\section{Remote Robot System with Force Feedback}

\subsection{System Configuration}

The configuration of the remote robot system with force feedback is shown in Figure 1. The system consists of two terminals called the master terminal and slave terminal. Each of the terminals consists of two PCs, and the PCs are connected 


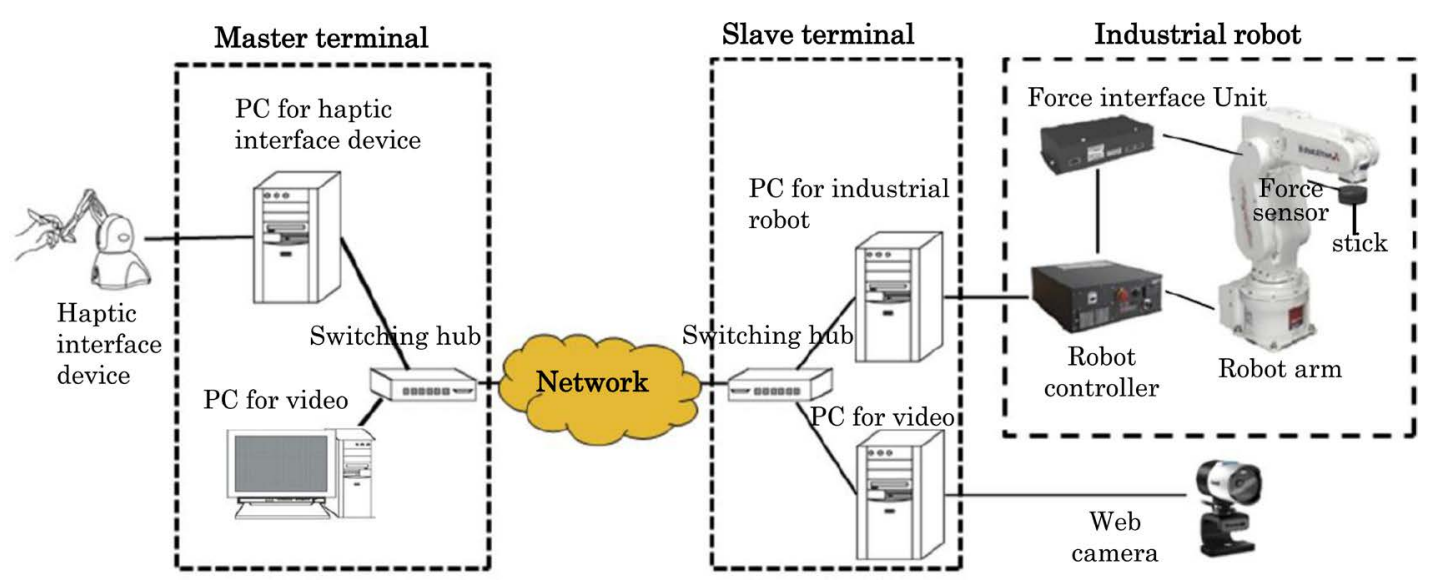

Figure 1. Configuration of remote robot control system with haptics.

to each other via a switching hub.

At the master terminal, a haptic interface device (Geomagic Touch [15]) is connected to PC for haptic interface device, and another PC is used for video. At the slave terminal, one of the two PCs is used for web camera (produced by Microsoft Corp., and video resolution is $1920 \times 1080$ pixels), and the other PC is used for industrial robot. The industrial robot consists of a robot arm (RV-2F-D by Mitsubisi Electric Corp. [16]), a robot controller (CR750-Q [16]), and a force sensor (1F-FS001-W200 [17]). The force sensor is attached to the surface of the flange of the robot arm. The force sensor is connected to the robot controller via the force interface unit. In order to investigate the effect of the proposed method, we handle objects (balls) with different types of softness in this paper.

\subsection{Remote Operation}

A user at the master terminal can operate the industrial robot at the slave terminal by using the haptic interface device while watching video (coding scheme: Motion JPEG, average bit rate: $4.5 \mathrm{Mbps}$ ). The default position of the haptic interface device is set to the origin, and the position corresponds to the default position of the industrial robot [9].

The master terminal updates the position information, calculates the reaction force, and outputs the reaction force every millisecond. The master terminal also transmits the position information to the slave terminal by User Datagram Protocol (UDP). At the slave terminal, the command information which is based on the position information received from the master terminal is sent to the industrial robot every 3.5 milliseconds by the real-time control function [18]. The force information is also acquired by the real-time control function, and the information is transmitted to the master terminal by UDP.

The reaction force $\boldsymbol{F}_{t}^{(m)}$ applied to the haptic interface device at time $t$ $(t \geq 1)$ is calculated as follows:

$$
\boldsymbol{F}_{t}^{(m)}=K_{\text {scale }} \boldsymbol{F}_{t-1}^{(s)}
$$

where $\boldsymbol{F}_{t-1}^{(s)}$ denotes the force receviced from the slave terminal, and $K_{\text {scale }}$ is a 
force scale which is set to 1 in this paper. Furthermore, since the maximum exertable force applied to the haptic interface device is $3.3 \mathrm{~N}$, the reaction force is set to $3.3 \mathrm{~N}$ when the calculated force is larger than $3.3 \mathrm{~N}$.

The position vector $S_{t}$ of the industrial robot outputted at the time $t(t \geq 2)$ is calculated as follows:

$$
\boldsymbol{S}_{t}=\left\{\begin{array}{l}
\boldsymbol{M}_{t-1},\left(\text { if }\left|\boldsymbol{M}_{t-1}-\boldsymbol{M}_{t-2}\right| \leq V_{\text {max }}\right) \\
\frac{V_{\text {max }}}{\left|\boldsymbol{M}_{t-1}\right|} \boldsymbol{M}_{t-1}, \text { (otherwise) }
\end{array}\right.
$$

where $\boldsymbol{M}_{t-1}$ is the position vector of haptic interface device received from the master terminal, and $V_{\max }$ is the maximum movement velocity. That is, in order to operate the robot arm safely, the maximum movement velocity is limited to $V_{\max }\left(V_{\max }=5 \mathrm{~mm} / \mathrm{s}\right.$ in this paper $)$.

\section{Stabilization Control}

\subsection{Conventional Method}

In bilateral control systems, unstability phenomena occur more easily as the network delay increases [6]. The reason is as follows: The master terminal calculates reaction force by using the force information received from the slave terminal, and updates the position based on the reaction force. The updated position is transmitted to the slave terminal and the slave terminal updates its position based on the position received from the master terminal. This means that there exists a mechanical closed loop. The phase delay of the loop increases when there exist network delays, and if the phase delay is larger than $180 \mathrm{deg}$, the system becomes unstable. This is an unpreventable problem when we use a network.

Therefore, one of solutions for the problem is to carry out stabilization control using the wave filter together with the phase control filter [6] which was previously proposed by the authors. The block diagram of the control is shown in Figure 2. In Figure 2, $G_{m}(s)$ is the transfer function of the master terminal, and $G_{s}(s)$ is that of the slave terminal; $f_{s}$ is the force information which is transmitted to the mater terminal from the slave terminal, and $f_{m}$ is the output force for the master terminal; $x_{m}$ is the position vector obtained at the master terminal, and $x_{r}$

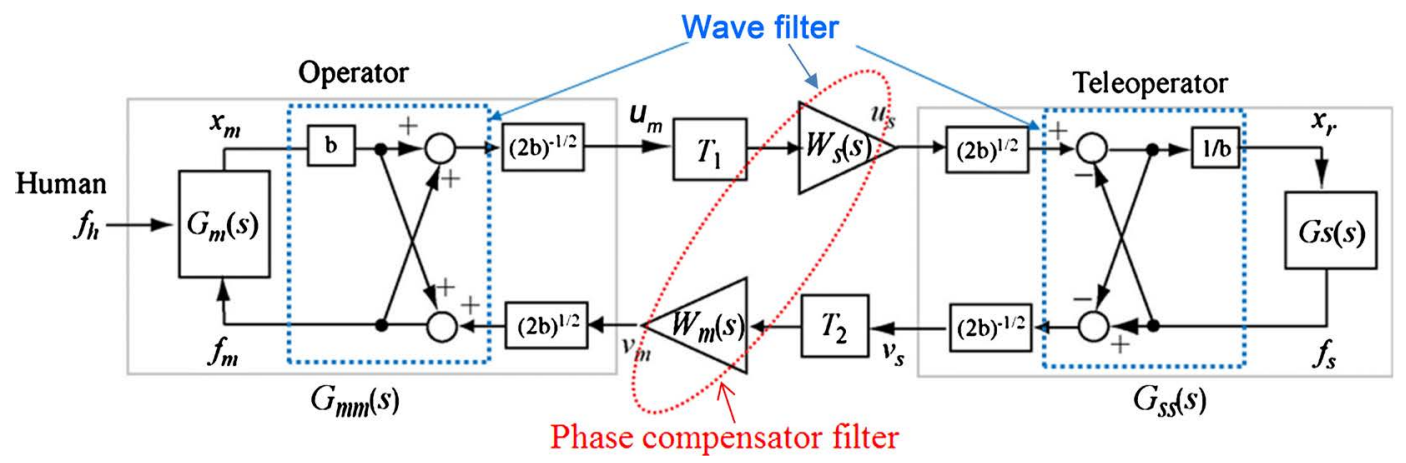

Figure 2. Block diagram of stabilization control. 
is the position vector for the slave terminal; $f_{h}$ is the force exerted from the user at the master terminal.

The wave filter includes the cross part and the coefficient $b$. The phase control filter includes $W_{s}(s)$ and $W_{m}(s)$. For further details, the reader is referred to [9] and [13].

\subsection{Enhancement Method}

In a preliminary experiment, when we apply the conventional method to the remote robot system in the three axes, unstability phenomena (vibrations of the robot and device) occured when users tried to push a hard object such as a tennis ball.

In order to solve the problem, we propose an enhancement method in which we incorporate a phase lead compensation filter $\left(\frac{1}{2} \times \frac{0.25 s+1}{0.08 s+1}\right)$ to $G_{m}(s)$ and a phase lag compensation filter $\left(\frac{1}{2} \times \frac{0.05 s+1}{0.25 s+1}\right)$ to $G_{s}(s)$. The former filter is used to solve the phase lag caused by the second order integration from the force to position. In order to avoid unstability due to the useless time of the industrial robot, the later filter is employed to lower the gain in high frequency ranges. Furthermore, $W_{s}(s)$ and $W_{m}(s)$ are set to $\frac{1}{(0.03 s+1)^{2}}$, and $b$ at the master terminal is set to 0.3 and that at slave terminal is set to 0.6 (this means that the mapping ratio [6] from the work space of the haptic interface device to that of the industrial robot is $2: 1$ ).

\section{Assessment Method}

In our experiment, we employ a network emulator (NIST Net [19]) instead of the network of Figure 1. A metal rod is fixed at the tip of the force sensor which is attached to the surface of the flange of the robot arm. Four fixtures are setting on the top of rack which is used to fix the industrial robot arm to fix objects as shown in Figure 3. We generate a constant delay (called the additional delay in this paper) for each packet transmitted between the master and slave terminals. We handle two types of work (work 1 and work 2) to investigate the effect of proposed method. In work 1, each subject is asked to push a ball by operating the robot arm while watching video, and in work 2 , the subject pushes a ball by operating the robot arm and identify what kind of the ball is pushed according to the softness without watching video. In the two types of work, the subject pushes the ball by the metal rod fixed at the robot arm.

In work 1, the subject pushes a ball from above and return back after pushing the ball for about 4 seconds, and he/she repeats the work three times (the subject is also asked to push the ball from the obliquely upward and the side, since the results are almost the same as those from above, we show only the results when the subjects push the ball from above). Because the effects of the conventional 


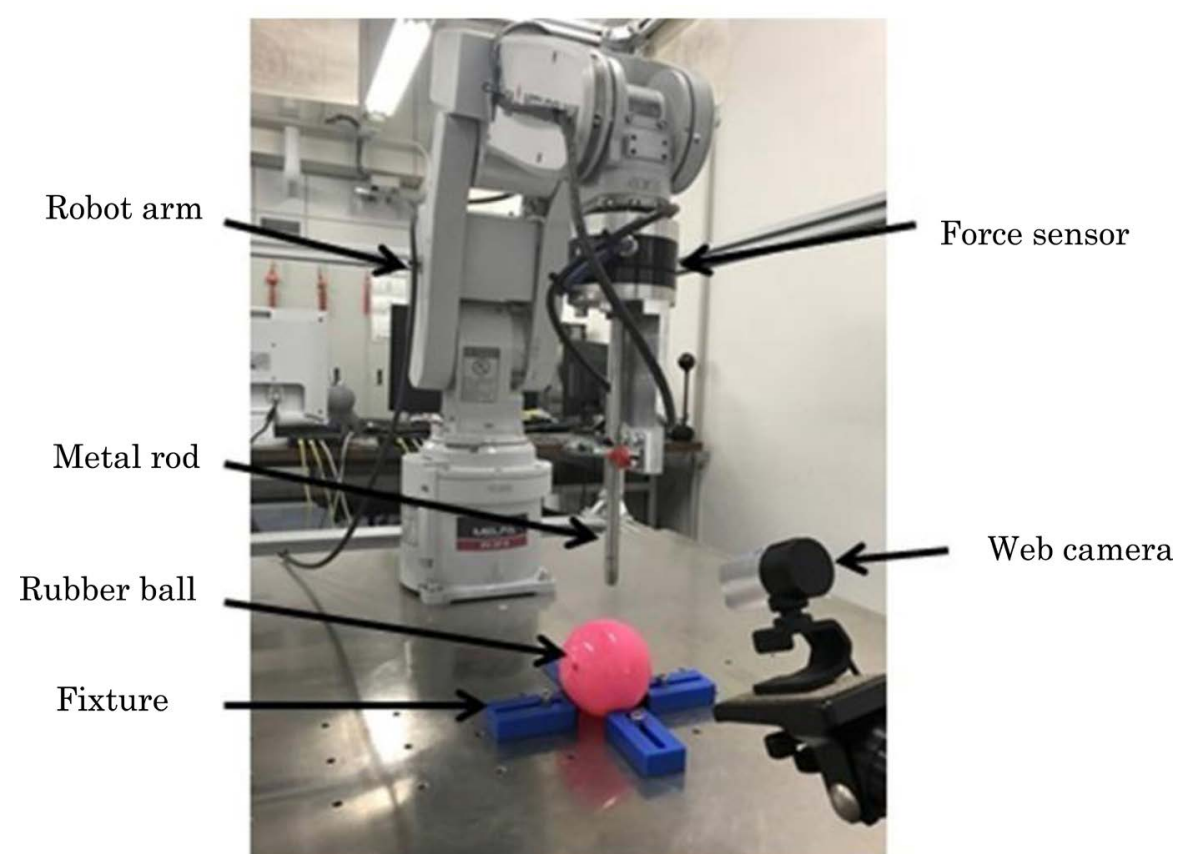

Figure 3. Appearance of robot arm.

method for soft objects have been confirmed, we here deal with only a tennis ball, which is used as a hard object in the work. In order to compare the proposed method with the conventional method, we carry out the assessment by changing the constant delay as follows: $0 \mathrm{~ms}, 100 \mathrm{~ms}, 200 \mathrm{~ms}, 400 \mathrm{~ms}$, and 800 ms. We measure the position of the haptic interface device, the position of the industrial robot, the output force of the haptic interface device, and the force obtained from the force sensor of the robot arm.

In work 2, we handle two balls with different softness as in [18]; one is a rubber ball (diameter: $70 \mathrm{~mm}$ ), and the other is a soft tennis ball (diameter: $65 \mathrm{~mm}$ ). Each subject pushes a ball from above and identifies the ball only by the softness he/she perceived. Therefore, we do not use the camera and the PCs for video in Figure 1. Furthermore, the robot arm is set to move only in vertical direction (the $y$ axis) so that all the subjects can do the work under the same condition [20]. Also, we use papers with thickness of $5 \mathrm{~mm}$ under the soft tennis ball so that the height of the two balls is the same.

Before the experiment, each subject practices to push the two balls to confirm the softness about 1 minute without any additional delay. In the practice, the type of ball is told to the subject, and then he/she tries to push a ball from above five times for each ball.

In the experiment, the subject pushed a ball as in the practice to identify the ball only by perceived softness without telling what the ball is. Each ball was selected 5 times, and the order was random for each subject. We carried out the experiment by setting the constant delay to $0 \mathrm{~ms}, 50 \mathrm{~ms}, 100 \mathrm{~ms}, 200 \mathrm{~ms}$, and $300 \mathrm{~ms}$, and obtained the average of correct answer rate. The number of subjects (males) whose ages were between 21 to 25 is 15 . 


\section{Assessment Results}

\subsection{Work 1 (Push Ball) (3 Axes)}

Experiment results of work to push a ball (tennis ball) are shown in Figure 4 through Figure 6. The results of the conventional method are shown in Figure 4, and those of the proposed method are shown in Figure 5. Since the system with the conventional method becomes unstable, we show only the results of the proposed method when the additional delay is $800 \mathrm{~ms}$ in Figure 6. Also, because the results in the $x$ and $z$ axes are almost the same as those in the $y$ axis, we demonstrate only the results in the $y$ axis.

From Figure 4, we see that the instability phenomena (vibrations) occur when the subject tries to push the ball. This means that the system is instable. From Figure 5, we find that there are almost no vibrations; we can say that the system is stable. However, the position of the haptic interface device is twice as that of the industrial robot. This is because the coefficient $b$ is set as described in 3.2.

From Figure 6, we can also notice that the system is stable as that in Figure 5. However, the position of the haptic interface device is delayed, and the delay is proportional to the network delay [8].

Furthermore, we carried out the experiment by using a soft tennis ball and a rubber ball, and we found that the stability can be attained by both conventional method and proposed method.

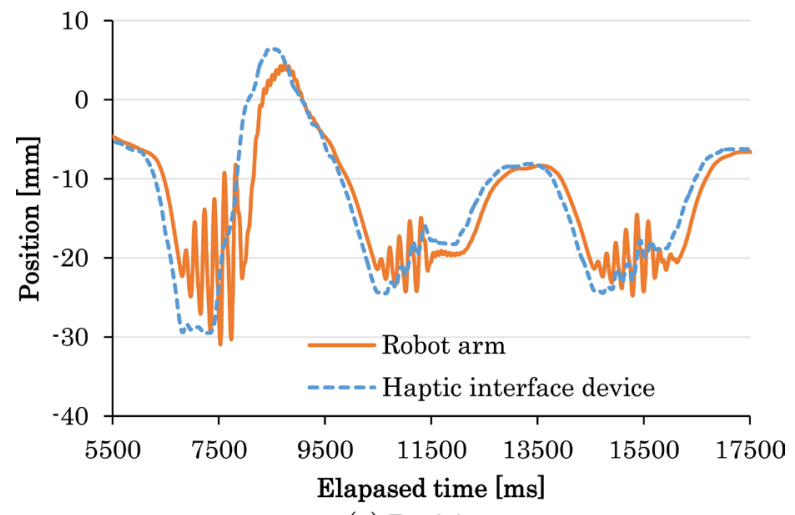

(a) Position

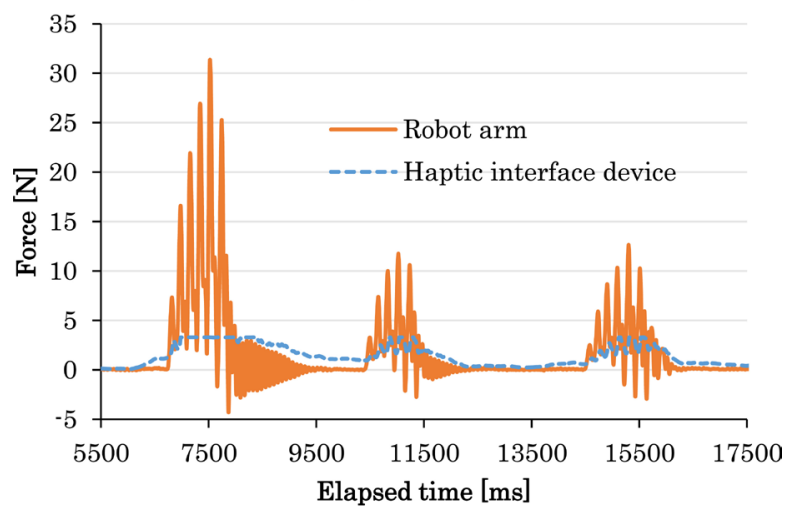

(b) Force

Figure 4. Position and force in conventional method ( $y$ axis, additional delay: $0 \mathrm{~ms}$ ). 


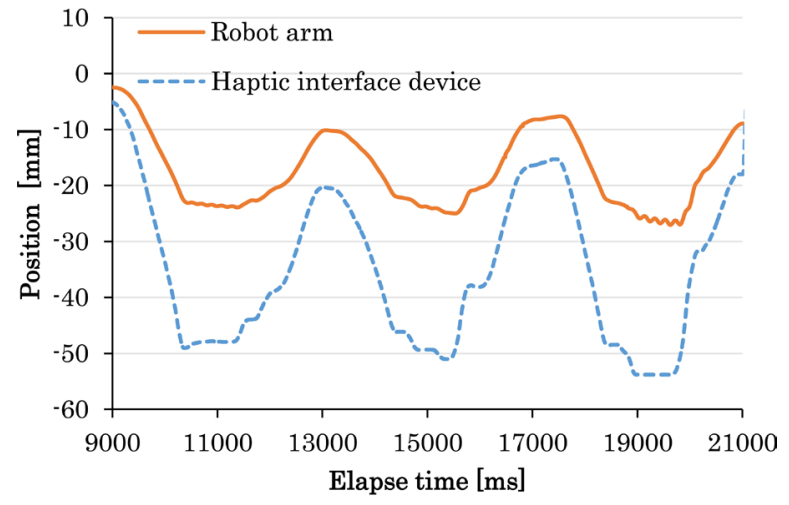

(a) Position

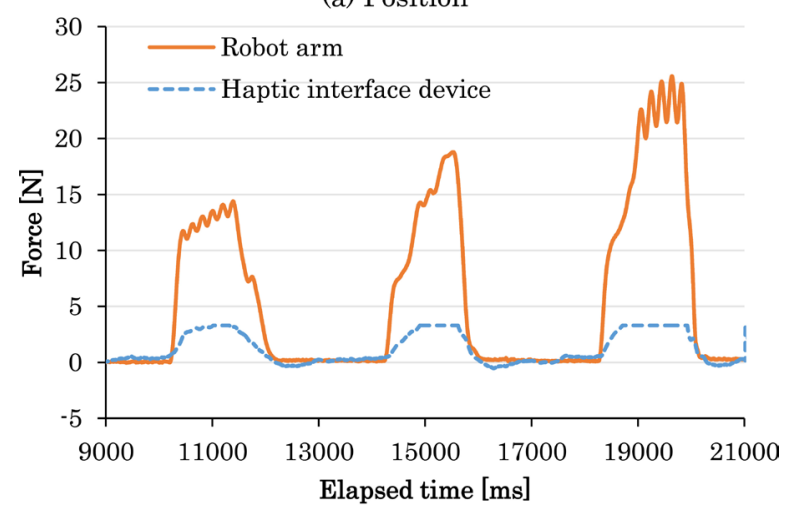

(b) Force

Figure 5. Position and force in proposed method ( $y$ axis, additional delay: $0 \mathrm{~ms}$ ).

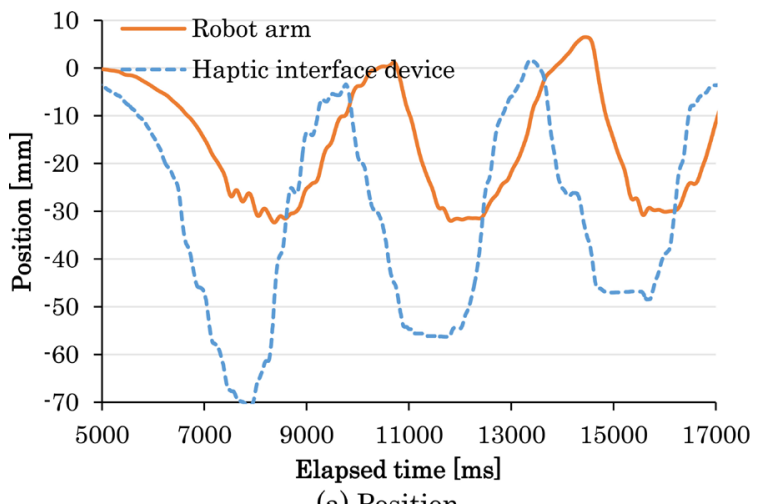

(a) Position

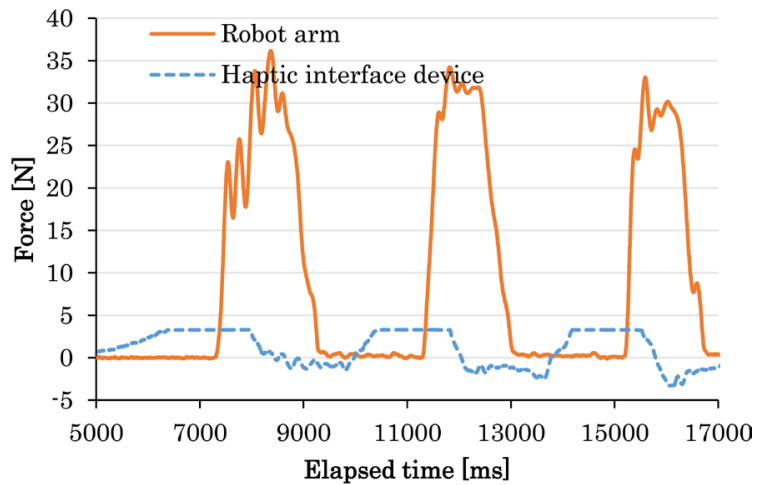

(b) Force

Figure 6. Position and force in proposed method (yaxis, additional delay: $800 \mathrm{~ms}$ ). 


\subsection{Work 2 (Ball Identification) (1 Axis)}

The average of correct answer rate versus the additional delay is shown in Figure 7. We also plot the $95 \%$ confidence intervals in the figure.

From Figure 7, we notice that the average of correct answer rate of the proposed method is better than that of the conventional method. In order to clarify the reasons, we compare the force of the haptic interface device and that of the robot arm in the proposed method with those in the conventional method. The results are shown in Figure 8 through Figure 11. From Figure 8 and Figure 9,

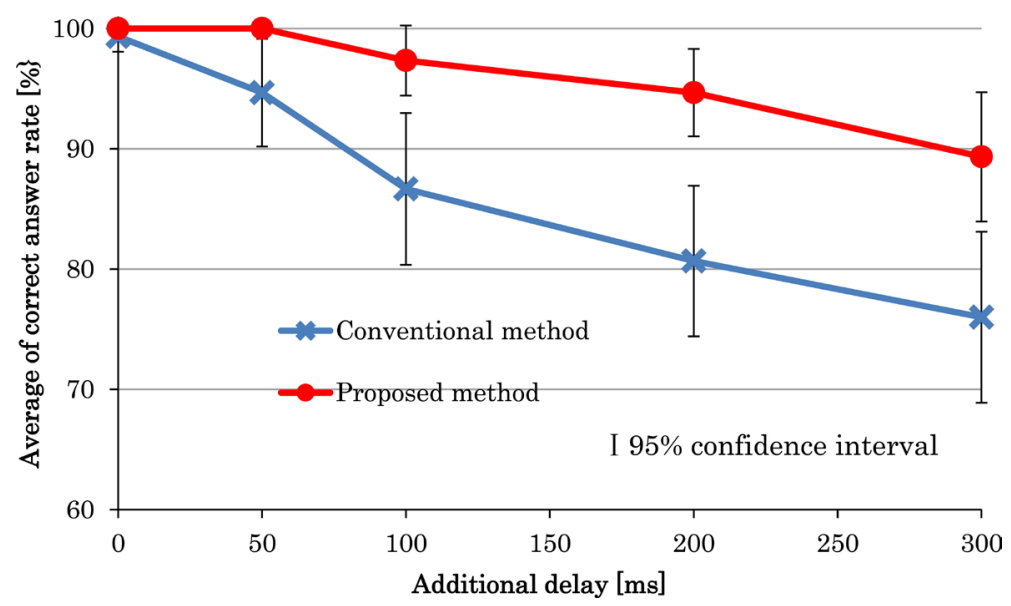

Figure 7. Average of correct answer rate versus additional delay.

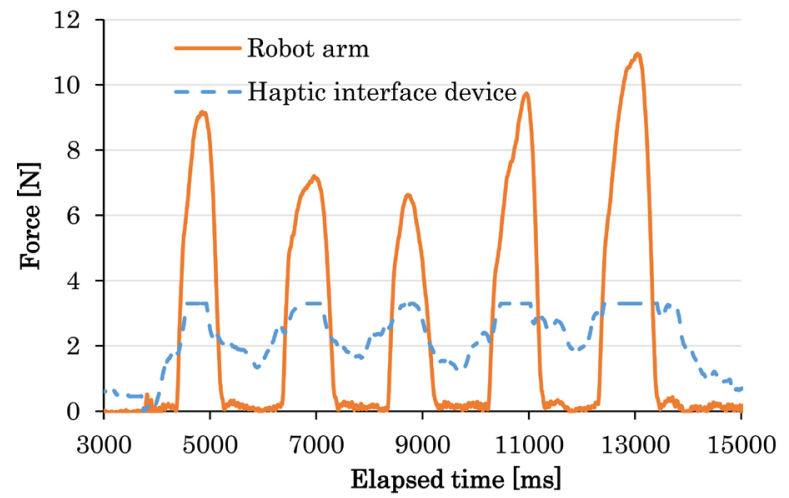

(a) Rubber ball

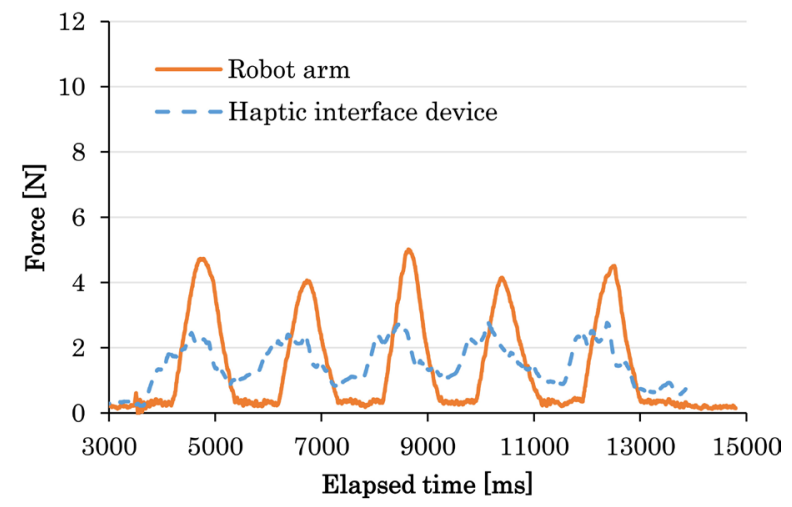

(b) Soft tennis ball

Figure 8. Force in conventional method (additional delay: $0 \mathrm{~ms}$ ). 


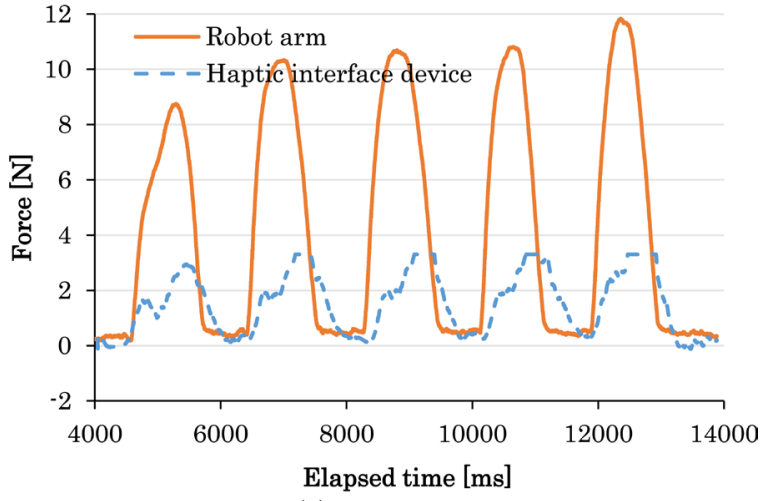

(a) Rubber ball

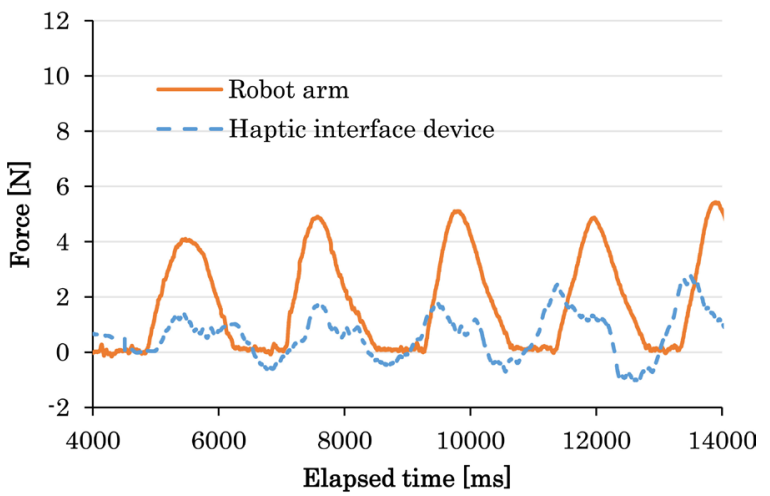

(b) Soft tennis ball

Figure 9. Force in proposed method (additional delay: $0 \mathrm{~ms}$ ).

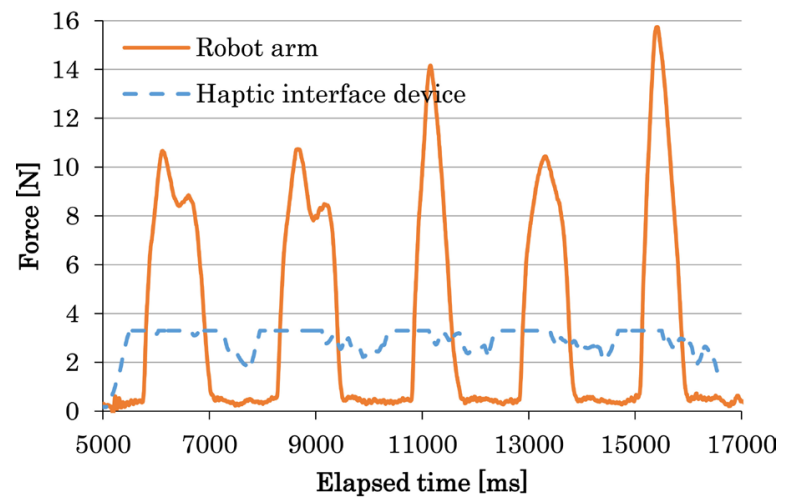

(a) Rubber ball

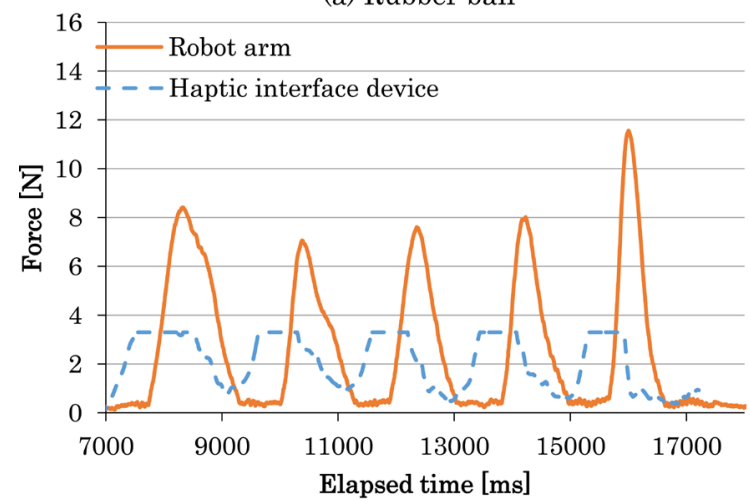

(b) Soft tennis ball

Figure 10. Force in conventional method (additional delay: $200 \mathrm{~ms}$ ). 


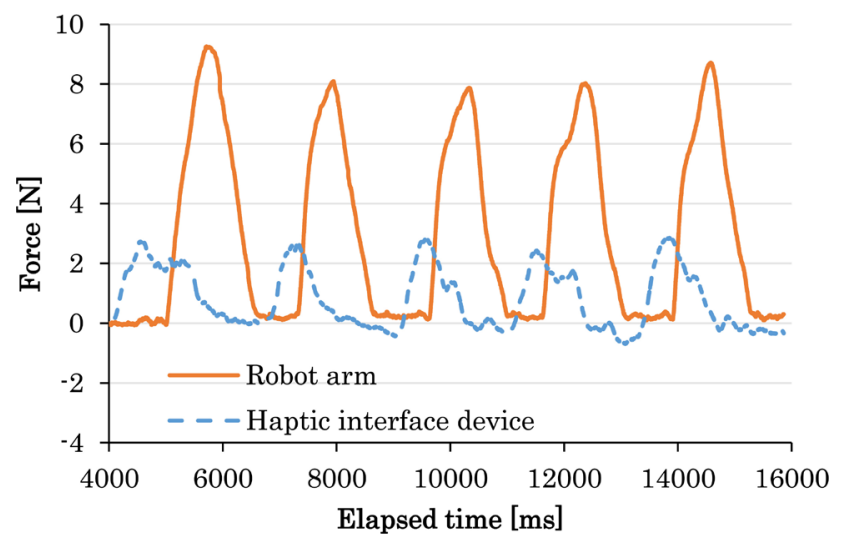

(a) Rubber ball

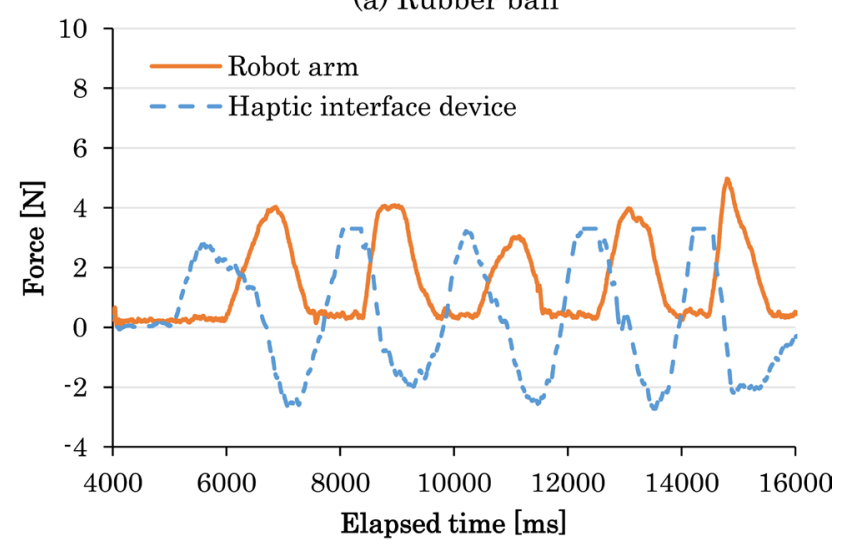

(b) Soft tennis ball

Figure 11. Force in proposed method (additional delay: $200 \mathrm{~ms}$ ).

when the additional delay is $0 \mathrm{~ms}$, we can see similar tendencies between the conventional method and the proposed method. Since the force of the haptic interface device and that of the robot arm are very different when the object is the rubber ball or the soft tennis ball, it is easy to identify the ball by the perceived softness, and the average of correct answer rate is almost 100\%. From Figure 10 and Figure 11, we find that the force of the haptic interface device and that of the robot arm are very different from each other when the balls are different in the proposed method. Therefore, the average of correct answer rate of the proposed method is higher than that of the conventional method. However, from Figure 10 and Figure 11, we see that when the object is the rubber ball, the force of the haptic interface device is more close to the real feeling of pushing the ball in the proposed method. However, when the object is the soft tennis ball, the force of the haptic interface device in the proposed method is more close to the real feeling of pushing the ball in the conventional method. In the proposed method, the subject felt larger reaction force which is opposite to the movement direction always, and it is difficult for the subject to perceive the softness of the ball.

From above discussion, we can say that the proposed method is more suitable for objects with certain hardness, and the conventional method has much better effects when the objects are not so hard. 


\section{Conclusions}

In this paper, we applied stabilization control using the wave filter together with the phase control filter which was previously proposed by the authors to the remote robot system with force feedback and proposed a method to enhance haptic quality. We carried out the experiment to investigate the effect of the proposed method and compare the effect with that of the conventional method. As a result, the proposed method is more suitable for objects with certain hardness, and the conventional method has much better effects when the objects are not so hard.

As the next step of our research, we need to clarify the effective domain by carrying out other types of work such as writing characters. It is also important to study QoS control and stabilization control together in multiple remote robot systems with force feedback.

\section{Acknowledgements}

The authors thank Yuichi Toyota and Eijirou Taguchi at Nagoya Institute of Technology for their support of the experiment. This work was supported by JSPS KAKENHI Grant Number 18K11261.

\section{Conflicts of Interest}

The authors declare no conflicts of interest regarding the publication of this paper.

\section{References}

[1] Ohnishi, K. (2013) Real World Haptics: Its Principle and Future Prospects. The Journal of the Institute of Electrical Engineers of Japan, 133, 268-269. (In Japanese) https://doi.org/10.1541/ieejournal.133.268

[2] Kawai, T. (2013) Haptics for Surgery. The Journal of the Institute of Electrical Engineers of Japan, 133, 282-285. https://doi.org/10.1541/ieejjournal.133.282

[3] Ishibashi, Y. and Huang, P. (2016) Improvement of QoS in Haptic Communication and Its Future. The IEICE Transactions on Communications, J99-B, 911-925. (Japanese Edition)

[4] Huang, P. and Ishibashi, Y. (2013) QoS Control and QoE Assessment in Multi-Sensory Communications with Haptics. The IEICE Transactions on Communications, E96-B, 392-403. https://doi.org/10.1587/transcom.E96.B.392

[5] ITU-T Rec. I. 350 (1993) General Aspects of Quality of Service and Network Performance in Digital Networks.

[6] Miyoshi, T., Maeda, Y., Morita, Y., Ishibashi, Y. and Terashima, K. (2014) Development of Haptic Network Game Based on Multi-Lateral Tele-Control Theory and Influence of Network Delay on QoE. Transactions of the Virtual Reality Society of Japan, Special Issues on Haptic Contents, 19, 559-569. (In Japanese)

[7] Huang, P., Miyoshi, T. and Ishibashi, Y. (2017) Stability Control in Remote Bilateral Robot Control System with Force Feedback. IEEE the 3rd International Conference on Control, Automation and Robotics, Nagoya, 22-24 April 2017.

[8] Huang, P., Miyoshi, T. and Ishibashi, Y. (2017) Stabilization of Bilateral Control in 
Remote Robot System. IEICE Technical Report, CQ2016-125. (In Japanese)

[9] Suzuki, K., Maeda, Y., Ishibashi, Y. and Fukushima, N. (2015) Improvement of Operability in Remote Robot Control with Force Feedback. 4th Global Conference on Consumer Electronics, Osaka, 27-30 October 2015, 16-20. https://doi.org/10.1109/GCCE.2015.7398509

[10] Banthia, V., Maddahi, Y., Zareinia, K., Liao, S., Olson, T., Fung, W., Balakrishnan, S. and Sepehri, N. (2017) A Prototype Telerobotic Platform for Live Transmission Line Maintenance: Review of Design and Development. Transactions of the Institute of Measurement and Control, 40, 3273-3292. https://doi.org/10.1177/0142331216687021

[11] ITU-T Rec. G. 100/P. 10 Amendment 1 (2007) New Appendix I: Definition of Quality of Experience (QoE).

[12] Matsunaga, K., Ishibashi, Y., Fukushima, N. and Sugawara, S. (2012) Effect of Adaptive Reaction Force Control in Remote Control System with Haptic Media and Video. The 27 th International Technical Conference on Circuits/Systems, Computers and Communications, Sapporo, 15-18 July 2002.

[13] Miyoshi, T., Terasima, K. and Buss, M. (2006) A Design Method of Wave Filter for Stabilizing Non-Passive Operating System. IEEE Conference on Computer Aided Control System Design, 2006 IEEE International Conference on Control Applications, 2006 IEEE International Symposium on Intelligent Control, Munich, 4-6 October 2006, 1318-1324. https://doi.org/10.1109/CCA.2006.286029

[14] Duong, M.D., Miyoshi, T., Terashima, K. and Rodriguezseda, E.J. (2008) Analysis and Design of Position-Force Teleoperation with Scattering Matrix. 17th IFAC World Congress, Seoul, 6-11 July 2008, 12715-12720. https://doi.org/10.3182/20080706-5-KR-1001.02151

[15] http://www.geomagic.com/en/products/phantom-omni/overview

[16] http://www.mitsubishielectric.co.jp/fa/products/rbt/robot/lineup/manual/f/bfp-a88 99k.pdf (In Japanese)

[17] http://dl.mitsubishielectric.co.jp/dl/fa/members/document/manual/robot/bfp-a8940 /bfp-a8940Z.pdf (In Japanese)

[18] http://dl.mitsubishielectric.co.jp/dl/fa/members/document/manual/robot/bfp-a8080 /bfp-a8080e.pdf (In Japanese)

[19] Carson, M. and Santay, D. (2003) NIST Net-A Linux-Based Network Emulation Tool. ACM SIGCOMM Computer Communication Review, 33, 111-126. https://doi.org/10.1145/956993.957007

[20] Taguchi, E., Ishibashi, Y. and Huang, P. (2017) Experiment on Softness Identification of Objects in Remote Robot System with Haptics: Effect of Stabilization Control. IEICE Technical Report, CQ2017-38. (In Japanese) 